\title{
The Tooth Contact Analysis of a Heavy-load Planetary Gearing
}

\author{
$\operatorname{Tan} \operatorname{Xin}^{1, a}$ \\ ${ }^{1}$ School of Electromechanical and Architectural Engineering, Jianghan University, Wuhan, Hubei, \\ China, 430056 \\ atanpatrick@163.com
}

Keywords: Planetary gearing; Tooth contact analysis; Modal analysis; ANSYS

Abstract. This paper presents a method for analyzing the tooth contact situations of a heavy-load planetary gearing based on Ansys Workbench. Firstly, a 3D finite element model of the whole gearing is established with 10 node tetrahedron elements. Then forces and loads bearing by the gearing are applied on the model to test the static strength. Some key parts of the gearing, such as planetary carriers and the sun gears, are specially checked. Finally, the stress concentration point of the engaging tooth of the second stage of the gearing is examined by the tooth contact analysis. The results show that the static strength is enough but the roots of the engaging tooth have higher stress than other areas.

\section{Introduction}

Being a sort of fossil fuel, petroleum is widely used. Thousands of ocean drilling platforms are mining petroleum around the world. Usually, an ocean drilling platform has three dozen multi-stage hybrid planetary gearings for its legs' elevating or dropping. These multi-stage hybrid planetary gearings have large speed reduction ratio and bear heavy loads. Their strength and dynamics behaviors have obvious impacts on the reliability of the whole machine.

Aided by the software Ansys Workbench, this article has established a 3D FE model of a multi-stage hybrid planetary gearing which is used for an ocean drilling platform. Based on simulation analysis, this paper studied the static strength and the tooth contact situations of the gearing.

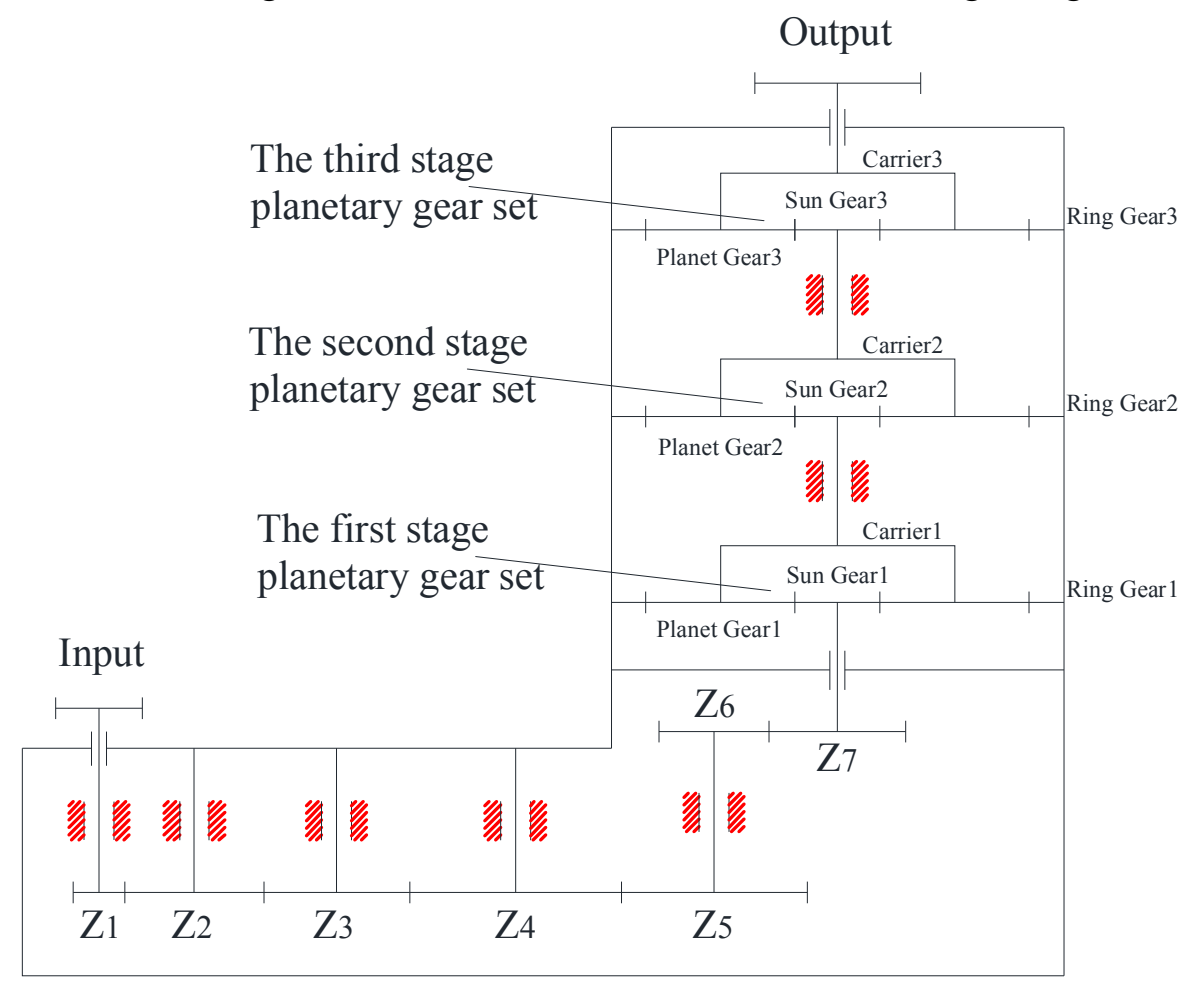

Fig. 1 The sketch of the multi-stage gearing 


\section{The FE model of the gearing}

The sketch of the multi-stage gearing is illustrated in Figure. 1. The 3D model of the gearing could be constructed in the software Pro/E and should be stored in an IGES file. The software Ansys Workbench provides data interfaces for exchanging IGES files or $\mathrm{X}_{-} \mathrm{T}$ files so that the 3D model of the gearing can be imported into Ansys Workbench. The flowchart is shown as Fig. 2.

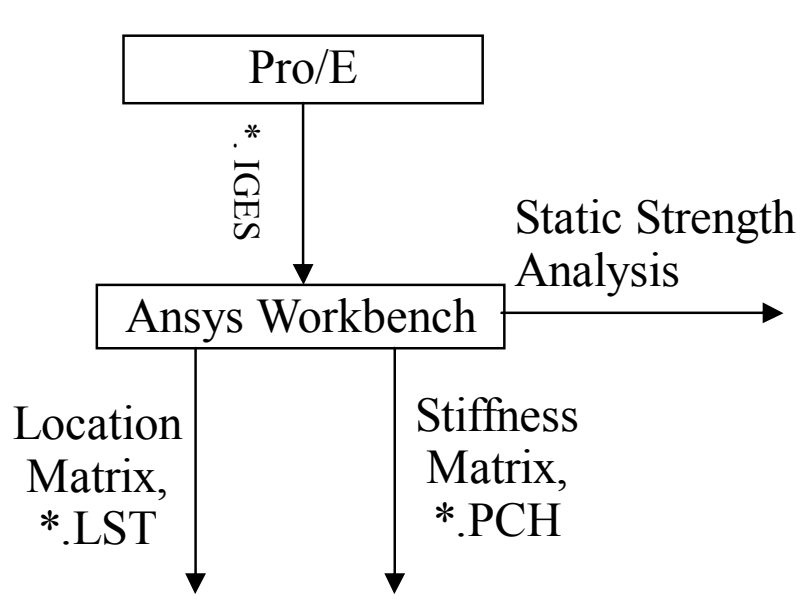

\section{Fig. 2 The flowchart of data exchanging}

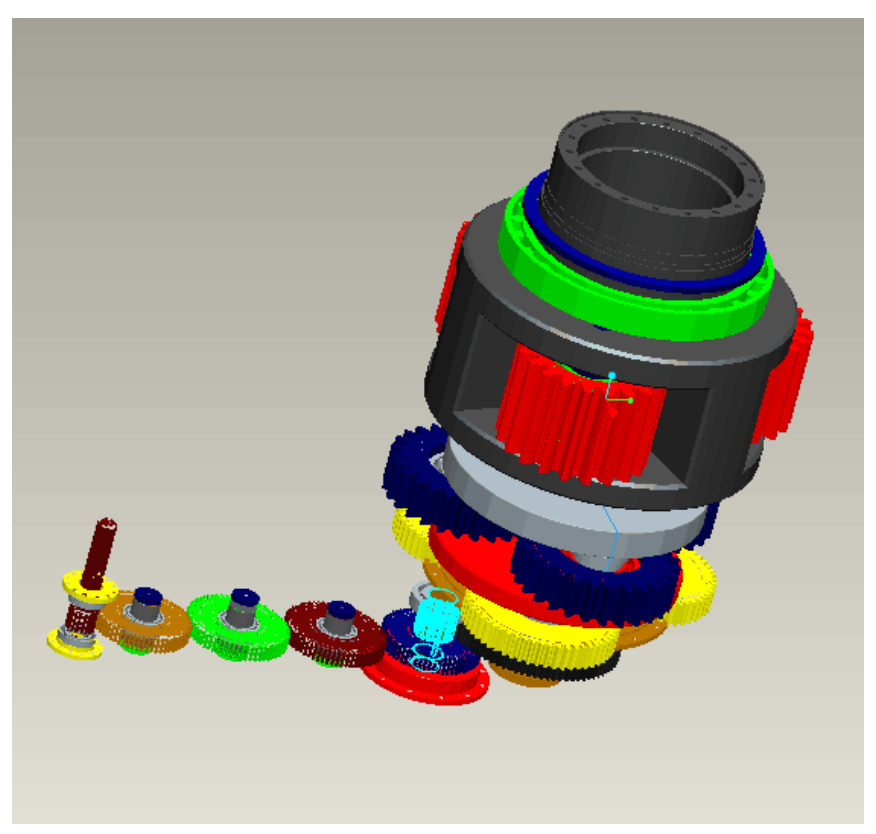

Fig. 3 The FE model of the gearing

In this paper, 10 nodes tetrahedron elements are used for generating the FE meshes of the whole gearing. And each pair of engaging tooth is considered as line-contacting. Each finite element on the contacting line is divided into two kinds of independent cells of stiffness $\mathrm{K}\left(\mathrm{M}_{\mathrm{i}}\right)$ and equivalent normal deviation $\delta_{\mathrm{e}}\left(\mathrm{M}_{\mathrm{i}}\right)$ to calculate tooth shape deviations. Fig. 3 shows the FE model of the gearing.

Planets are assimilated to rigid cylinders characterized by screws of infinitesimal displacements whose co-ordinates in the frame fixed to the sun-gear/planet $j$ centre-line are ${ }^{[1]}$

$$
\left\{\tau_{j}\right\}=\left\{\begin{array}{l}
u_{j}^{R}\left(O_{j}\right)=v_{j} \stackrel{r}{S}_{j}+w_{j} \stackrel{r}{T}_{j}+u_{j}{ }_{j}{ }_{j} \\
r \underset{r}{\omega}{ }_{j}=\varphi{ }_{j}+\psi_{j}{ }_{j}+\theta_{j}{ }_{j}{ }_{j}
\end{array}\right.
$$

where (1) $\mathrm{O}_{\mathrm{j}}$ denotes centre of planet $\mathrm{j}$;

(2) $S_{j}, T_{j}, Z_{j}$ are the unit vectors of the reference frame attached to planet $j$;

(3) $\mathrm{v}_{\mathrm{j}}, \mathrm{w}_{\mathrm{j}}, \mathrm{u}_{\mathrm{j}}$ are the translational degrees of freedom;

(4) $\varphi_{\mathrm{j}}, \psi_{\mathrm{j}}, \theta_{\mathrm{j}}$ are the rotational degrees of freedom.

\section{The tooth contact analysis}

The aim of the tooth contact analysis is to determine points at which the highest stress occurred. The software Ansys Workbench provides 3 ways to analysis the tooth contact situations: ${ }^{[2]}$

(1) Point-Point Contact: this method applies especially to situations that slippage is small or nonexistent. In this case, the position of the contact point should be found before analyzing.

(2) Line-Line Contact: this method applies to the situations that the precise position of the contact point is not sure.

(3) Surface-Surface Contact: in this case, two contacting surfaces are considered as flexible surfaces and the deformation is only described on the radial direction. 
Because the position of contact points is not known, this paper uses the second way to analyze tooth contact situations.

Before tooth contact analyzing, it is necessary to determine working loads, constraint forces and parameters used in Ansys Workbench. The whole gearing is made of steels (20CrMnTi) whose mechanical properties are shown in Fig. 4.

- Structural
\begin{tabular}{|l|c|}
\hline P Young's Modulus & $2.06 e+011$ \\
\hline P Poisson's Ratio & 0.29 \\
\hline P Density & 7850. \\
\hline$\square$ Thermal Expansion & $1.2 \mathrm{e}-005$ \\
\hline$\square$ Alternating Stress \\
\hline$\square$ Strain-Life Parameters \\
\hline$\square$ Tensile Yield Strength & $2.5 e+008 \mathrm{~Pa}$ \\
\hline$\square$ Compressive Yield Strength & $2.5 e+008 \mathrm{~Pa}$ \\
\hline$\square$ Tensile Ulimate Strength & $4.6 e+008 \mathrm{~Pa}$ \\
\hline$\square$ Compressive Utimate Strength & $0 . \mathrm{Pa}$ \\
\hline
\end{tabular}

Fig. 4 Mechanical properties of 20CrMnTi

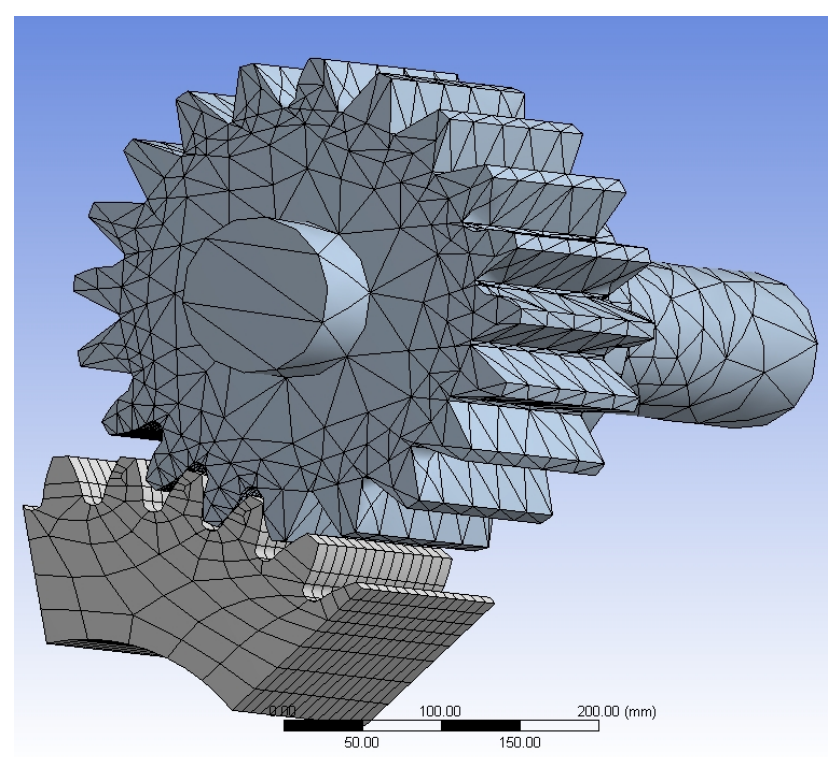

Fig. 5 The FE meshes of engaging tooth

Ansys Workbench can automatically generate FE meshes for users with the unique density. But some areas of gears, such as tooth, are more important than other areas, such as flanks, so that gears' tooth should have denser meshes than others ${ }^{[3]}$. Fig. 5 shows the FE meshes of engaging tooth of the second stage of the gearing. Working loads exerted on the engaging tooth can be calculated according to the power and torque they transmitted. The results of the tooth contact analysis are shown in Fig. 6 .

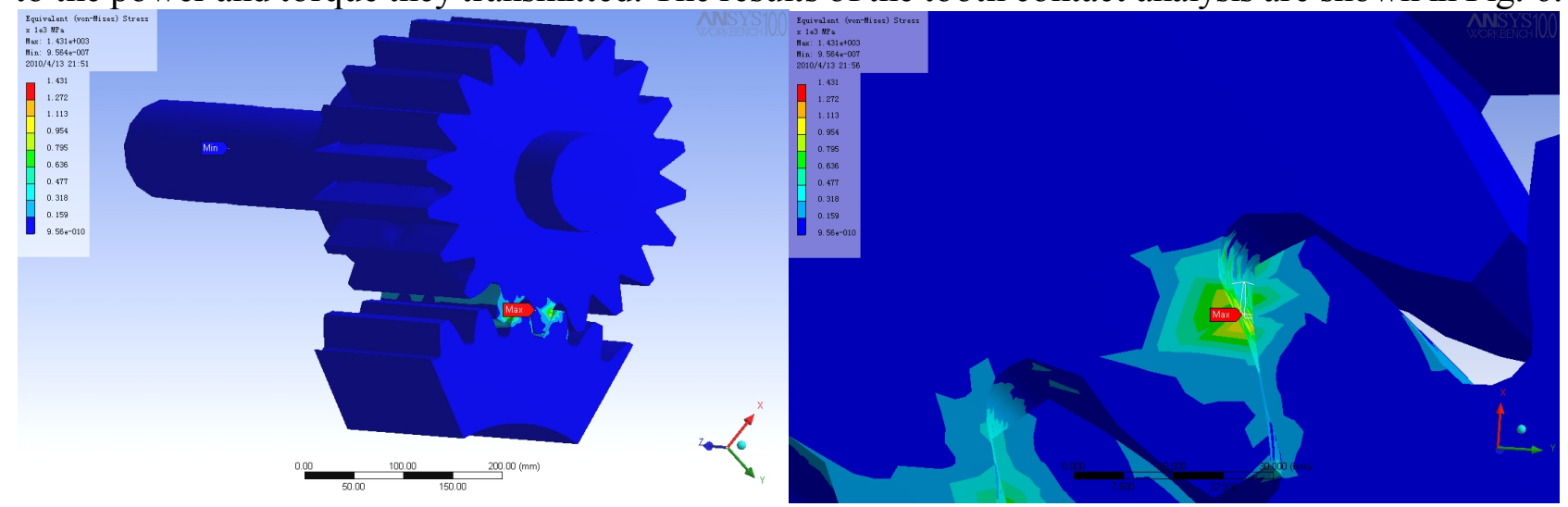

Fig. 6 Von Mise stress of engaging tooth of the second stage of the gearing

The point which bears the maximum contact stress appears on the root of the pinion. The maximum value is $\sigma_{\mathrm{Pmax}}=137.58 \mathrm{MPa}$ (for the pinion) or $\sigma_{\mathrm{Wmax}}=106.93 \mathrm{MPa}$ (for the wheel). It is obvious that stresses exerted on the bottom of a pinion's teeth are much higher than that of other areas of the gearing. Accordingly, the maximum deformation reaches $3.59 \mathrm{e}-3 \mathrm{~mm}$ while the minimum strain is $5.78 \mathrm{e}-4 \mathrm{~mm}$. All data indicates that the gearing should have larger module and more tooth.

\section{Modal analysis of the shaft}

Modal analysis of the shaft aims to explore inherent frequencies and vibration modes of the shaft because of the shaft's dynamic behaviors take much effect on the dynamics of the whole gearing. The 
algorithm for modal analysis in this paper is the Block Lanczos algorithm ${ }^{[4,5]}$ due to its faster convergent speed and more accurate calculation. Fig. 7 shows the FE model of the shaft.

The first vibration mode of the shaft is a bending - lateral modal whose frequency is $2819.9 \mathrm{~Hz}$ (See Fig. 8). The second vibration mode (See Fig. 9) is an expanding elastic modal whose frequency is $3670.9 \mathrm{~Hz}$. Because the frequencies of the first two modal are high, we can get a conclusion that the shaft is too thick and heavy.

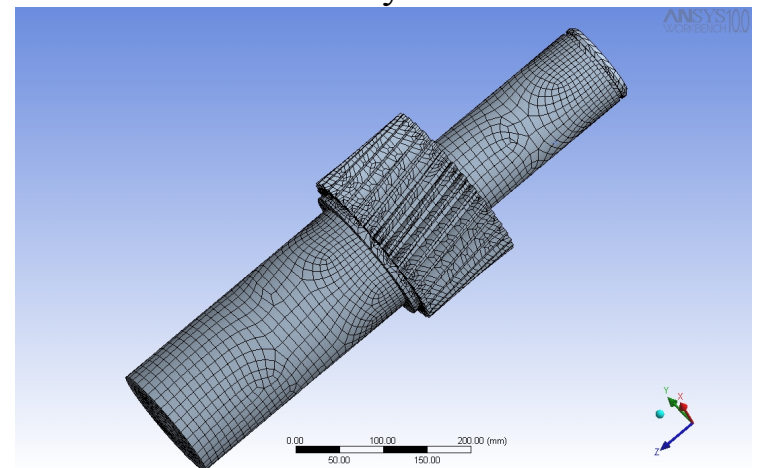

Fig. 7 The FE model of the shaft

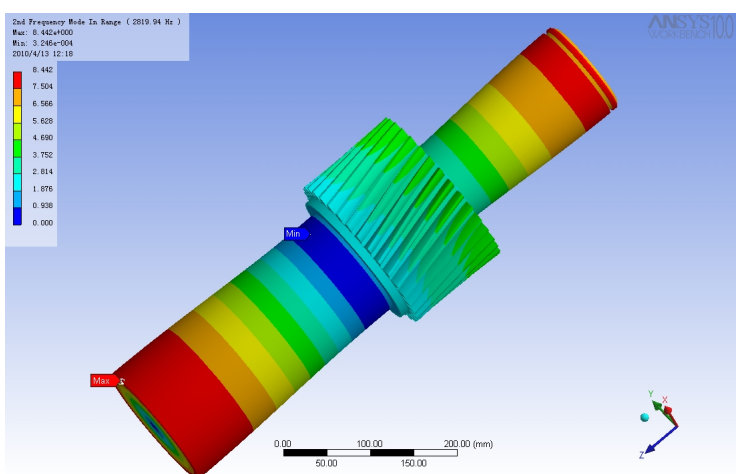

Fig. 8 The first modal of the shaft

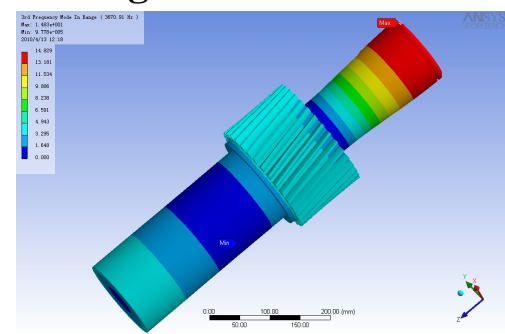

Fig. 9 The second modal

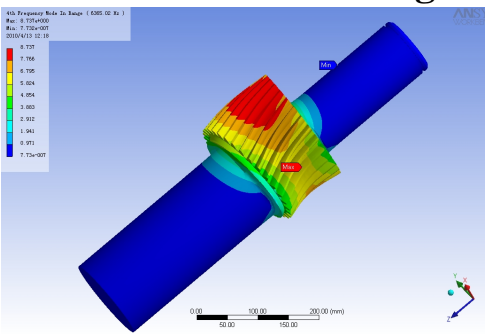

Fig. 10 The third modal

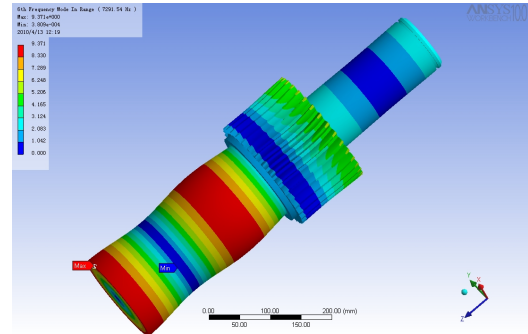

Fig. 11 The forth modal

The third and forth modal of the shaft (shown in Fig. 10 and Fig. 11) are abundant vibration modes whose amplitudes are large and the displacements are obvious.

\section{Conclusions}

This paper introduces a methodology for establishing the FE model of a multi-stage hybrid gearing used in ocean drilling platforms. Based on the software Ansys Workbench, the tooth contact analysis are applied to the second stage of the gearing. The results show that the contact stresses distributed on the tooth's surfaces are high so that the module and tooth number of the gearing should be modified. Dynamics analyses show that frequencies of the first two vibrations are high. The shaft should be lightened.

Acknowledgements: This paper is sponsored by Wuhan Education Bureau.

\section{References}

[1] Kousaku. OHNO. Naoyuki. TANAK. A. Contact Stress Analysis for Helical Gear with 3-Dimensional Finite Elenment Method[M]. 2001:1-7.

[2] Edri, R; AI. Nais, M.O. Prestressed modal analysis using finite element package ANSYS[J]. Third International Conference On Numerical Analysis and Its Applications, NAA 2004.

[3] V. Abousleiman, P. Velex, "A hybrid 3D finite element/lumped parameter model for quasi-static and dynamic analyses of planetary/epicyclic gear sets", in Journal of Mechanism and Machine Theory, pp. 725-748

[4] A. Kahraman, "Natural-modes of planetary gear trains", in Journal of Sound and Vibration, 173 (1) (1994), pp. 125-130. 
[5] T.-J. Yeh, Feng-kun Wu, "Modeling and Robust Control of Worm-gear Driven Systems", in Proceedings of the 2005 IEEE International Conference on Mechatronics, 2005, pp. 711-716. 